\title{
Application of the Mathieu's equation for a design of a photonic crystal supporting surface electromagnetic waves
}

\author{
Ekaterina Rostova, Giovanni Dietler, and Sergey K. Sekatskii
}

\author{
Laboratoire de Physique de la Matière Vivante, IPSB, BSP, \\ Ecole Polytechnique Fédérale de Lausanne (EPFL), CH-1015 Lausanne, Switzerland \\ *corresponding author: ekaterina.rostova@epfl.ch
}

\begin{abstract}
Nowadays, unique characteristics of surface electromagnetic waves, particularly, surface plasmons supported by a specially designed photonic crystal find numerous applications. We propose to exploit an evident analogy between such a photonic crystal and a structure with a sine-modulated refractive index. The light propagation inside the latter is described by the famous Mathieu's differential equation. This application of the Mathieu's equation can be useful for a design of multilayer structures, and also for fundamental understanding of electromagnetic phenomena in inhomogeneous media.
\end{abstract}

\section{Introduction}

Photonic crystals (PCs) are materials that possess a periodic modulation of their refractive indices (RIs) on the scale of the light wavelength [1]. Optical surface modes in onedimensional photonic crystals (multilayer dielectric mirrors) were extensively studied in the 1970s, both theoretically [2, 3] and experimentally [4]. Twenty years later, excitation of optical surface waves (SW) in photonic crystals in a Kretschmann-like configuration was first demonstrated [5]. These studies rapidly led to broad use of surface waves based on photonic crystals in ever-widening applications in the field of optical sensors [6-14]. This technique benefits much from its unique peculiarities, namely, the existence of both $p$ - and $s$-polarized surface waves enables to discriminate surface and volume effects. Moreover, an electromagnetic field penetration depth into an external medium is significantly increased compared to surface plasmon polaritons (SPR)-based biosensors. Surface waves-based biosensors permit to study not only interactions between relatively thin layers of proteins (and other biomolecules), but also between such thick objects as bacteria, cells and cell organelles. A direct experimental comparison of the sensitivity of biosensors based either on surface plasmon polaritons or on photonic crystal surface waves (same as Bloch waves or Tamm states) may be found elsewhere [15]. The authors showed that a biosensor based on photonic crystal surface waves can demonstrate almost two times higher sensitivity that one based on surface plasmon polaritons.

Quite an important particular application of photonic crystals is their use to support long-range surface plasmon polaritons existing in such structures as photonic crystal thin metal layer - infinite dielectric [16] (of course, such electromagnetic waves exist only for $p$-polarized incident light [17]). These systems have much in common with well-known symmetric sandwich structures such as a thin metal layer between two infinite dielectrics, known to support long-range surface plasmons (from now on, when this cannot lead to a confusion, we will omit the word "polariton" and speak simply about plasmons) $[18,19]$. For this case the photonic crystal can be characterized, for a certain wavelength, by the same refractive index as that of an infinite dielectric, thus forming a symmetric sandwich structure. Exploitation of such structures enabled to excite and use in practice surface plasmons in thin $\operatorname{Pd}[12,20]$ and Co (to be submitted) layers as well as blue (at $405 \mathrm{~nm}$ ) [21] and UV (at $375 \mathrm{~nm}$; to be submitted) plasmons in thin gold layers. Note that if a photonic crystal is not exploited, for all these cases there are no reasons to speak about plasmons at all, because their propagation length is just in the order of a light wavelength.

All these circumstances well underline the necessity of deep and physically transparent understanding of the details of plasmon propagation in the structures involving photonic crystals. Certainly, rather effective calculation methods, based essentially on multiple reflections inside the multilayer structure (Fresnel formulae), do exist and are successfully used; see e.g. Konopsky's paper [22] which, in our opinion, is one of the best examples. Still we believe that these methods are rather formal and do not clearly reveal the physics of the phenomenon beyond. As a consequence, they are not indeed "intuition-friendly" and, correspondingly, their prediction potential without detailed calculations rests limiting.

In this work, we use an evident analogy between a one-dimensional photonic crystal and a structure with a sine-modulated refractive index. The propagation of light inside the latter is described by the famous Mathieu's differential equation, which extensive studies of many years had a considerable impact on the common knowledge, see e.g. a classic book [23] and numerous references therein. Strictly speaking, a photonic crystal (Fig. 1), which is a periodic sequence of dielectric layer pairs of thicknesses $d_{L}$ and $d_{S}$ with respectively large $n_{L}=\sqrt{\varepsilon_{L}}$ and small $n_{S}=\sqrt{\varepsilon_{S}}$ refractive indices, is not exactly a medium with a sine-modulated refractive index. Similar situation exists, for example, for electrons in crystals: planes of ions do create a periodic potential but do not create a sine potential. 
Nevertheless, this circumstance does not make an application of the Mathieu's equation for the corresponding problem meaningless. Rather the contrary, the same equation appears as the tool to tackle related problems. However, unfortunately the paradigm of the Mathieu's equation and its application are not commonly used to describe problems related to excitation of optical modes. By the present study we aim to broad applications of this equation and introduce it to the area of investigation into photonic crystals. Briefly, the present article discusses application of the Mathieu's differential equation to design a photonic crystal with a metal film supporting long-range surface modes.

The article is divided into five chapters: first, we introduce the Mathieu's equation and explain how an electromagnetic field distribution in periodic structures can be calculated by solving the equation. We emphasize on the requirements on its solutions which must be fulfilled to describe the surface modes. Second, we give numerical examples of photonic crystals with certain parameters. The given examples illustrate how to design a photonic crystal to excite surface waves at a desired wavelength. Next, solutions of the Mathieu's equation are analyzed to demonstrate physical limits of the optical properties of the periodic structures for surface wave excitation. In conclusion, an analogy was drawn between surface waves and energy eigenstates for a quantum particle in a periodic potential.

\section{Mathieu's equation}

Below we consider a system infinite dielectric - finite metal layer - infinite periodic dielectric structure with a sine profile of the refractive index (PC), see Fig. 1. Let the infinite dielectric has a dielectric constant $\varepsilon_{1}$ and metal$\varepsilon_{m}$, while a dielectric constant of the periodic structure is given by $\varepsilon(z)=\varepsilon_{a v}+\Delta \varepsilon \cos (2 \pi z / L)$, where $L$ is the period of the structure, and for the moment we identify an average dielectric constant with $\varepsilon_{a v}=\left(\varepsilon_{L}+\varepsilon_{S}\right) / 2$, $\Delta \varepsilon=\varepsilon_{a v}-\varepsilon_{S}=\varepsilon_{L}-\varepsilon_{a v}(\Delta \varepsilon>0)$. A finite-thickness layer characterized by the dielectric constant $\varepsilon_{m}$ should not be necessarily a metal; we will use this notation just to simplify the terminology.

Based on the properties of surface plasmons, which are "transverse magnetic (TM) in nature" and on known properties of TM electromagnetic waves [24], one can write for the $H_{y}$ - component of the magnetic field [19]:

$$
\vec{H}=H_{0} \vec{e}_{y} f(z) \exp (i \omega t-i \beta x),
$$

where $\vec{e}_{y}$ is a unit vector along the $y$-axis (Fig. 1). For TM modes, if we know the $H_{y}$ - component of the magnetic field, the electric field components can be evaluated from Maxwell's equations [2, 19]:

$$
E_{x}=\frac{i}{\omega \varepsilon_{0} \varepsilon} \frac{\partial H_{y}}{\partial z}, E_{z}=-\frac{\beta}{\omega \varepsilon_{0} \varepsilon} H_{y} .
$$

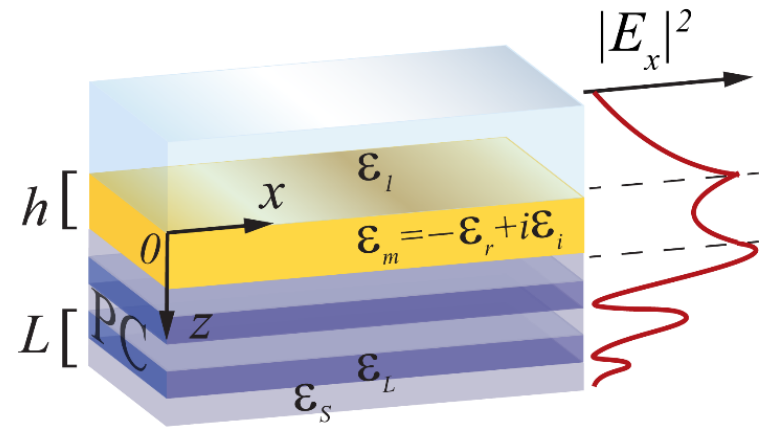

Figure 1. A schematic of the studied structure (not to scale): dielectric $(\mathrm{z}<0)-$ metal $(0<\mathrm{z}<\mathrm{h})-$ photonic crystal $(\mathrm{z}>\mathrm{h})$. The dielectric constants are $\varepsilon_{1}, \varepsilon_{m}, \varepsilon_{S}$ and $\varepsilon_{L}$, respectively; the last two correspond to the dielectic constants of the alternating layers of the photonic crystal with the period $L$. The bold red line depicts the electric field intensity distribution of a surface wave in the structure, the dashed line images the metal layer boundary. The surface wave propagates along the $\mathrm{x}$-axis.

The $H_{y}$ - components of electromagnetic field inside the homogeneous media are given by solutions of appropriate wave equations, which in our case reduce to

$$
\frac{\partial^{2} H_{y}}{\partial z^{2}}+\left(k^{2} \varepsilon_{1, m}-\beta^{2}\right) H_{y}=0
$$

where we introduce $k^{2}=\varepsilon_{0} \omega^{2}=(2 \pi / \lambda)^{2}$.

The equations (2) and continuity of the tangential $H_{y^{-}}$ field component at the interfaces $z=0, z=h$ together with the continuity of the tangential $E_{x}$-field component at the interface $z=0$ yield the following solution of the wave equation:

- $\quad$ Inside an infinite dielectric, $z<0, f_{1}(z)=e^{S_{1} z}$ (this implies $\operatorname{Re}\left(\mathrm{S}_{1}\right)>0$; exactly this form of a solution describes the surface wave, i.e. a wave localized at the interface),

- Inside the metal, $0<z<h$, we have $f_{m}(z)=\cosh \left(S_{m} z\right)+\frac{S_{1} \varepsilon_{m}}{S_{m} \varepsilon_{1}} \sinh \left(S_{m} z\right)$,

- Inside the medium with a varying refractive index (PC), $z>h$,

$f_{3}(z)=\left(\cosh \left(S_{m} h\right)+\frac{S_{1} \varepsilon_{m}}{S_{m} \varepsilon_{1}} \sinh \left(S_{m} h\right)\right) P(z-h)$,

where $P(z)$ is an appropriate solution of the Mathieu's equation (see eq. (6) below). Here the exponential decay constant is given by

$$
S_{1, m}^{2}=\beta^{2}-\varepsilon_{1, m} k^{2} .
$$

The solution of the Mathieu's equation is chosen to exponentially decrease for $z \rightarrow+\infty$, and $P(0)=1$, which ensures also the continuity of $H_{y}$ on the interface $z=h$. The continuity of $E_{x}$ at the interface $z=h$ will be established later on when a solution of the Mathieu's equation will be discussed. 
Inside a medium with a varying refractive index (PC), the wave equation reduces to the Mathieu's equation

$$
\frac{d^{2} H_{y}}{d z^{2}}-\left(\beta^{2}-\varepsilon_{a v} k^{2}-\Delta \varepsilon k^{2} \cos (2 \pi z / L)\right) H_{y}=0 .
$$

With the variable change $u=\pi z / L$, eq. (5) yields a standard form of the Mathieu's equation [23] which will be in the main focus of the present study:

$$
\frac{d^{2} H_{y}}{d u^{2}}+(a-2 q \cos (2 u)) H_{y}=0 .
$$

Here $a$ and $q$ are parameters given by

$$
\begin{gathered}
a=-\beta^{2} L^{2} \pi^{-2}+\varepsilon_{a v} k^{2} L^{2} \pi^{-2}, \\
q=-\frac{1}{2} \Delta \varepsilon k^{2} L^{2} \pi^{-2} .
\end{gathered}
$$

\section{Surface electromagnetic waves}

To obtain surface waves in the studied structure, besides a requirement to satisfy the continuity of the $E_{x^{-}}$ field component at $z=h$, a solution $y(u)$ of the Mathieu's equation should exponentially decrease for $u \rightarrow+\infty$. The exponentially decreasing solutions belong to the instability regions of $(a, q)$-chart of the Mathieu's equation parameters (hatched areas on Fig. 2, adapted from Ref. 23), which are associated with photonic band gaps.

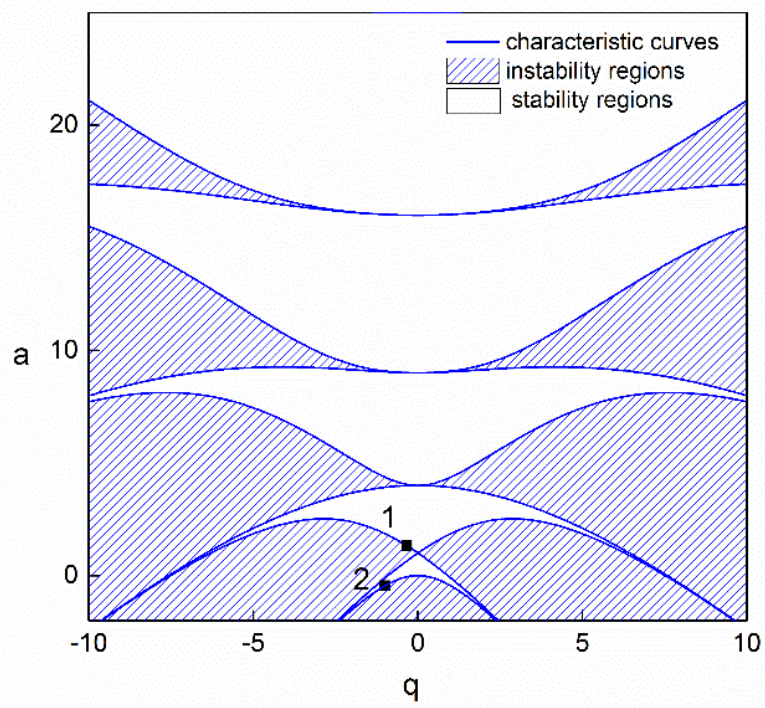

Figure 2. The stability chart for the solutions of the Mathieu's equation with the parameters $(a, q)$. The solid lines correspond to characteristic curves, the hatched areas depict the instability regions, the empty areas - stability regions. The solutions marked by the points ' 1 ' and ' 2 ' are analyzed in the text.

Let us now analyze the condition of the continuity of $E_{x}$-field component on the metal - PC interface. From now on we denote the variable as $u=\pi(z-h) / L$ to place the interface at $u=0$. The aforementioned continuity condition reads:

$$
\begin{aligned}
& \frac{S_{m}}{\varepsilon_{m}} \sinh \left(S_{m} h\right)+\frac{S_{1}}{\varepsilon_{1}} \cosh \left(S_{m} h\right)= \\
& =\frac{1}{\varepsilon_{3}(0)}\left(\cosh \left(S_{m} h\right)+\frac{S_{1} \varepsilon_{m}}{S_{m} \varepsilon_{1}} \sinh \left(S_{m} h\right)\right) \frac{\pi}{L} y^{\prime}(0),
\end{aligned}
$$

where $\varepsilon_{3}(0)$ is a local, just near the interface, value of the photonic crystal dielectric constant.

It is natural to start further analysis of propagation modes in our system using an analogy to long-range surface plasmons in symmetric sandwich structures, in which a photonic crystal is replaced by a semi-infinite uniform dielectric with the dielectric constant $\varepsilon_{3}=\varepsilon_{1}$. In a thin metal film with $h \rightarrow 0$, the long-range surface plasmons exist if $S_{1}=S_{3}=0[18,19]$, which is necessary to ensure the continuity of the longitudinal electric field component at the interface. This means, see (3), that the propagation constant is given by $\beta=\sqrt{\varepsilon_{1}} k$, so that from (7) we obtain the photonic crystal period

$$
L=\frac{\lambda \sqrt{a}}{2 \sqrt{\varepsilon_{a v}-\varepsilon_{1}}} .
$$

Next, the continuity condition (9) results in $y^{\prime}(0)=0$, and such a solution $y(u)$ can be constructed as follows. The Mathieu's equation describing a magnetic field inside a periodic multilayer structure (photonic crystal) is given by eq. (6) which we rewrite as

$$
\frac{d^{2} y}{d u^{2}}+(a-2 q \cos (2 u)) y=0,
$$

with the following relation between the parameters $a$ and $q$ :

$$
q=-\frac{1}{2} \Delta \varepsilon \frac{a}{\left(\varepsilon_{a v}-\varepsilon_{1}\right)} .
$$

The exponentially decreasing solution of the equation (11) has the form of $y(u)=e^{-\mu u} \varphi(u)$, where $\mu>0$ is a characteristic exponent and $\varphi(u)$ is a $2 \pi$-or $\pi$-periodic function. The continuity condition of the magnetic field $H_{y}$ at the interface $u=0$ requires that $\varphi(0)=1$; such a solution exists [23] and can be found. To find it, the easiest numerical method, called the "shooting method", supposes to vary the value of $b$ in the initial condition $y^{\prime}(0)=b$ while solving numerically the ordinary differential equation (11), and to analyze the behavior of the solution for large $u$ : only one certain value of $b$ corresponds to an exponentially decaying solution. If at certain value of $u_{0}$ the derivative $\left.y^{\prime}(u)\right|_{u=u_{0}}=0$, then all that we need to construct a solution which gives rise to surface waves, is to shift the variable $u$ and consider the function $y_{1}(u)=C e^{-\mu\left(u+u_{0}\right)} \varphi\left(u+u_{0}\right)$; normalization constant $C$ is so chosen that $y_{1}(0)=1$. The function

$f_{3}(z)=\left(\cosh \left(S_{m} h\right)+\frac{S_{1} \varepsilon_{m}}{S_{m} \varepsilon_{1}} \sinh \left(S_{m} h\right)\right) y_{1}(\pi(z-h) / L)$ then describes the $H_{y}$-component of the electromagnetic field inside the photonic crystal. 
This simple consideration quite clearly demonstrates the physical meaning of a photonic crystal design supporting long-range surface plasmons. The expression (10) corresponds to an optimal selection of the thicknesses of the alternating layers constituting the photonic crystal, while the aforementioned shift of the initial value of the variable $u$ is nothing else than the necessity to add an additional (of large or small refractive index) layer with the well-defined thickness to ensure such propagation [22]. Moreover, we clearly see also additional possibilities which appear for photonic crystal-based structures but are lacking for symmetric sandwich structures. For the case of photonic crystals, the condition $S_{I}=0$ is not any more necessary: even without it, the boundary condition (9) which (again in the limit $h \rightarrow 0$ ) reads

$$
\frac{S_{1}}{\varepsilon_{1}}=\frac{1}{\varepsilon_{3}(0)} \frac{\pi}{L} y_{1}^{\prime}(0),
$$

still can be fulfilled. Remember that $S_{1}$ is necessarily positive, hence we need $y_{1}{ }^{\prime}(0)>0$ at the interface: just after the metal - PC interface, the absolute value of electromagnetic field vector should increase (of course, for larger distances it will eventually decrease according to its exponential decay when $u \rightarrow \infty)$. This corresponds to stronger localization and deeper penetration of the electromagnetic field inside transparent non-absorbing medium (photonic crystal) and consequently its weaker localization inside a lossy metal. As a result, a smaller fraction of the electromagnetic field energy is dissipated in the metal thus leading to even larger propagation distances of the surface waves (compare again with Ref. 22). Note, however, that if $S_{1} \neq 0$, also $L \neq \frac{\lambda \sqrt{a}}{2 \sqrt{\varepsilon_{a v}-\varepsilon_{1}}}$ but should be recalculated from eq. (7) with the given values of $S_{1}$ and $a$ : this shows certain subtlety of the task to optimize corresponding structures. This case will be discussed in details elsewhere.

\section{Numerical examples}

In the previous sections we showed how the Mathieu's equation can be applied to design a multilayer structure which supports long-range surface plasmons. Such surface modes can be excited in the structure which design and physical properties correspond to a pair of $(a, q)$-parameters lying in the unstable region on the stability chart (Fig. 2). These parameters, according to eq. (7), immediately define a pair of $L, \beta$ values and also the $S_{l}, S_{m}$ values using eq. (4).

As a starting point, we will study numerically the properties of the solutions in the first unstable region of negative $q$ and positive $a$. We chose the parameter $a$ close to 1 and parameter $q$ such that the pair $(a, q)$ is very close to the characteristic curve separating stability and instability regions (but, of course, still belongs to the instability region; see Fig. 2, point ' 1 '). For such an (a, q)-pair, according to the known properties of the Mathieu's equation, its vanishing at infinity solution is approximately given by $y_{1}(u) \cong e^{-\mu u} c e_{1}(u, q)$. Here the index $\mu$ is much less than one (see Ref. 23 Fig. 11) and $c e_{1}(u, q)$ is the Mathieu function describing a $2 \pi$-periodic solution of the Mathieu's equation for the pair $(a, q)$ lying exactly on the corresponding characteristic curve. This curve, when $a$ is slightly larger than one and $q$ small, is described by the following expansion (eq. (17), of Ref. 23):

$$
a=1+q-q^{2} / 8-q^{3} / 64-q^{4} / 1536+11 q^{5} / 36864+O\left(q^{6}\right) .
$$

The above $y_{1}(u)$ solution is clearly a complete analogue of the symmetric sandwich case, in which in the external medium, the magnetic field amplitude is described by $f_{3}(z)=C e^{-S_{3} z}$ with $S_{3}$ very close to zero: both these solutions decrease away from the interface very slowly. The further the chosen $(a, q)$-pair from the characteristic curves on the chart is, the larger is the difference of our solution from one for the symmetric sandwich case: we may call these solutions "more unstable and less periodic".

Let us now illustrate the consideration given above by several numerical calculations corresponding to real photonic crystal structures. For the case $S_{I}=0$, the equations (8) and (10) fix the ratio of the parameters

$$
\frac{q}{a}=-\frac{1}{2} \frac{\Delta \varepsilon}{\varepsilon_{a v}-\varepsilon_{1}} .
$$

Suppose we would like to optimize a structure which supports the surface waves at the wavelength of $739 \mathrm{~nm}$. We assume that $n_{S}=1.455, n_{L}=2.076$ (Ref. 22, such a photonic crystal has been quite successfully used to support long-range surface plasmons in $\operatorname{Pd}$ films $[12,20])$ so that $\varepsilon_{a v}=3.2134, \Delta \varepsilon=1.0964$ and $q / a=-0.2477$ using eq. (15). For the parameter $a$ slightly larger than 1, this ratio defines $q=-0.333$ and $a=1.344$ on the characteristic curve, and the optimal period of the photonic crystal $L=288$ $\mathrm{nm}$ calculated from eq. (10). To approximately describe the electromagnetic field inside the photonic crystal, we can use the following $q$-expansion of the $c e_{1}(u, q)$ function (p. 21 of Ref. 23):

$$
\begin{aligned}
c e_{1}(u, q)=\cos u- & \frac{q}{8}\left(1+\frac{q}{8}+\frac{q^{2}}{192}+\ldots\right) \cos (3 u)+ \\
& +\frac{q^{2}}{192}\left(1+\frac{q}{6}+\frac{q^{2}}{128}+\ldots\right) \cos (5 u)+\ldots
\end{aligned}
$$

It is immediately clear that $c e_{1}{ }^{\prime}(0, q)=0$ hence this function exactly corresponds to the boundary condition we are looking for: the photonic crystal refractive index just at the interface is equal to $n_{L}$. Analogically, for $a$ slightly smaller than one belonging to the characteristic curve (Fig. $2)$, the magnetic field inside a photonic crystal will be close to the function $s e_{1}(u, q)$ (see its $q$-expansion at p.13 of Ref. $23)$. The continuity condition of $\operatorname{se}_{1}{ }^{\prime}(\pi / 2, q)=0$ is fulfilled hence the refractive index of the photonic crystal right at the interface should be equal to $\sqrt{\varepsilon_{a v}}$.

Now, we will look at those decreasing to zero solutions in the proximity of $a=1$ which lie between two 
characteristic curves (Fig. 3). We are interested in the question either for all values of $a$ one may find such a solution $y(u)$ which at certain points satisfies the condition $y_{1}{ }^{\prime}(u)=0$, or there are such values of $a$ that always $y_{1}{ }^{\prime}(u)<0$. The latter would mean that no surface electromagnetic waves for such parameters $a, q$ exist. Numerical results attest that in the close proximity of $a=1$ (i.e. for small q) between two characteristic curves, the solution and its derivative are almost periodic (Fig. 4, graphs 1-2). Similar situation occurs when $q$ is approaching -6 , so that the pair $(a, q)$ again lies very close to the characteristic curve. The further the point on the chart from the characteristic curves is, the faster the solution is decreasing. This means that the derivative of such a decreasing solution is mainly negative, however the numerical results show that the derivative is positive at certain intervals (and, correspondingly, is equal to zero at some points, Fig. 4 graphs 3-5). We have checked a number of pairs $(a, q)$ belonging to the line of $q / a=-0.25=$ const (Fig. 3, Fig. 4 graph 1), and for all of them the points where $y_{1}{ }^{\prime}(u)=0$ were found.

Next, we analyzed the points described by the ratio $q / a=-1.5$ and lying deeper in the instability region. For all the chosen points on this line, we succeeded to find such initial conditions that the derivative was equal to zero at certain points. Moreover, the chosen points belonging to the lines $a=1.1=$ const, $q=3=$ const, and several other randomly chosen points were still suitable to find the solutions giving rise to the surface waves, though these points lie quite far away from the characteristic curves (Fig. 3, Fig. 4 graphs 3-5). Summarizing, despite the analyzed points were chosen in different locations in the region of positive parameter $a$ and negative $q$, we could not find any that would correspond to a decreasing at infinity solution with a derivative never equals to zero.
Other instability regions of Mathieu equation which correspond to larger values of $a$, such as $a=4$, 9, etc. [23], also give rise to surface waves which can be considered quite similarly. These solutions are an analogue of larger order resonances in Fresnel reflection of light inside a PC (increase of the relative phase differences on $2 \pi n$-values) and do not seem very interesting.

Now let us note another possibility. The above analysis suggests that we should still be able to construct a photonic crystal supporting long-range plasmons even if an external medium is such that $\varepsilon_{1}$ is larger than $\varepsilon_{a v}$. In such a case, from eq. (7) we see that $a<0$; still having at our disposal a certain finite value of $\Delta \varepsilon$ (and hence of $q$ ) we are able to find such a pair $(a, q)$ that the derivative of the corresponding function $y^{\prime}(u)=0$ for some values of $u$. For this, we again should work not too far from the curve separating unstable region of $a<0$ and small $q$ from a narrow stable region existing for larger values of $q$ (see Fig. 2 and Ref. 23). For this characteristic curve, under the condition of a negative $a$ close to zero, we have (p. 15 of Ref. 23):

$$
a=-q^{2} / 2+7 q^{4} / 128-29 q^{6} / 2304+O\left(q^{8}\right) .
$$

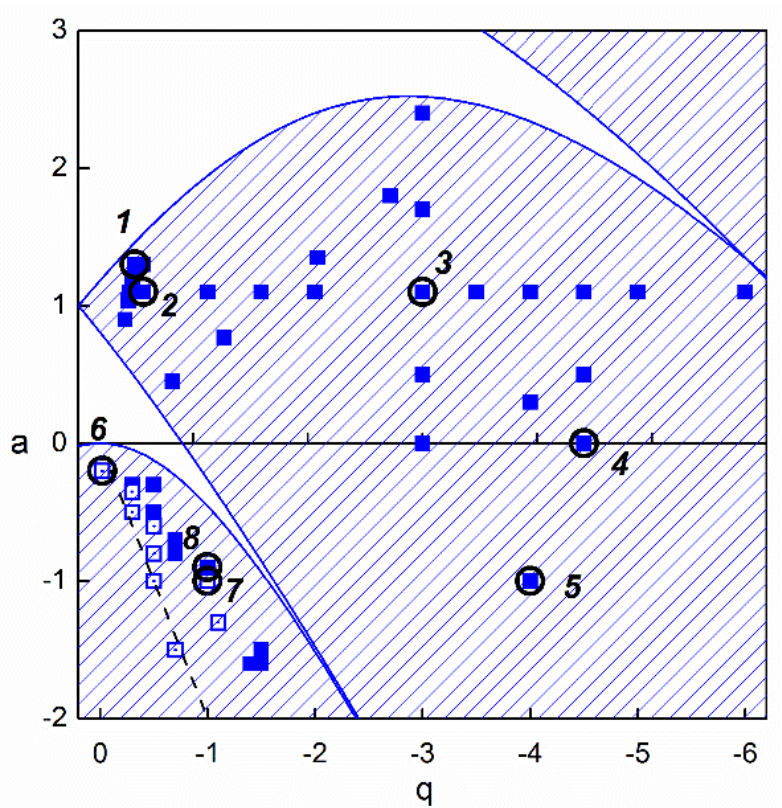

Figure 3. The points of $(a, q)$-diagram corresponding to the analyzed solutions of the Mathieu's equation in the instability region (hatched area). Depending on the solution behavior, it may describe surface modes (filled squares) or be inappropriate because of the impossibility to fulfill the continuity condition (empty squares). The black numbered empty circles denote $(a, q)$-pairs for which the solution and its derivative are plotted below (Fig. 4). The dashed black line corresponds to $\mathrm{a}=-2 \mathrm{q}$ (see explanation in the text).

Let us illustrate this possibility taking the same wavelength of $739 \mathrm{~nm}$ and $q=-1$. Then $q / a=2.184$ (Fig. 2, point 
'2') which gives $n_{1}=1.86$ and, the necessary period $L=\frac{\lambda \sqrt{-a}}{2 \sqrt{\varepsilon_{1}-\varepsilon_{a v}}}=1092 \mathrm{~nm}$. Under these $(a, q)$-parameters, the magnetic field component $H_{y}$ inside the photonic crystal is approximately described by $\pi$ - periodic function $c e_{0}(u, q)$ which satisfies the condition $c e^{\prime}{ }_{0}(0, q)=0$ (p. 49 of Ref. 23).
In this part of the chart, we again tried to find $(a, q)$ pairs that would correspond to such decaying solution for which always $y_{1}{ }^{\prime}(u)<0$, and we succeeded to find many of them. Indeed, we observed a tendency that for the majority of $|a|>|q|$, a solution that can describe surface modes was not found by varying the initial conditions.

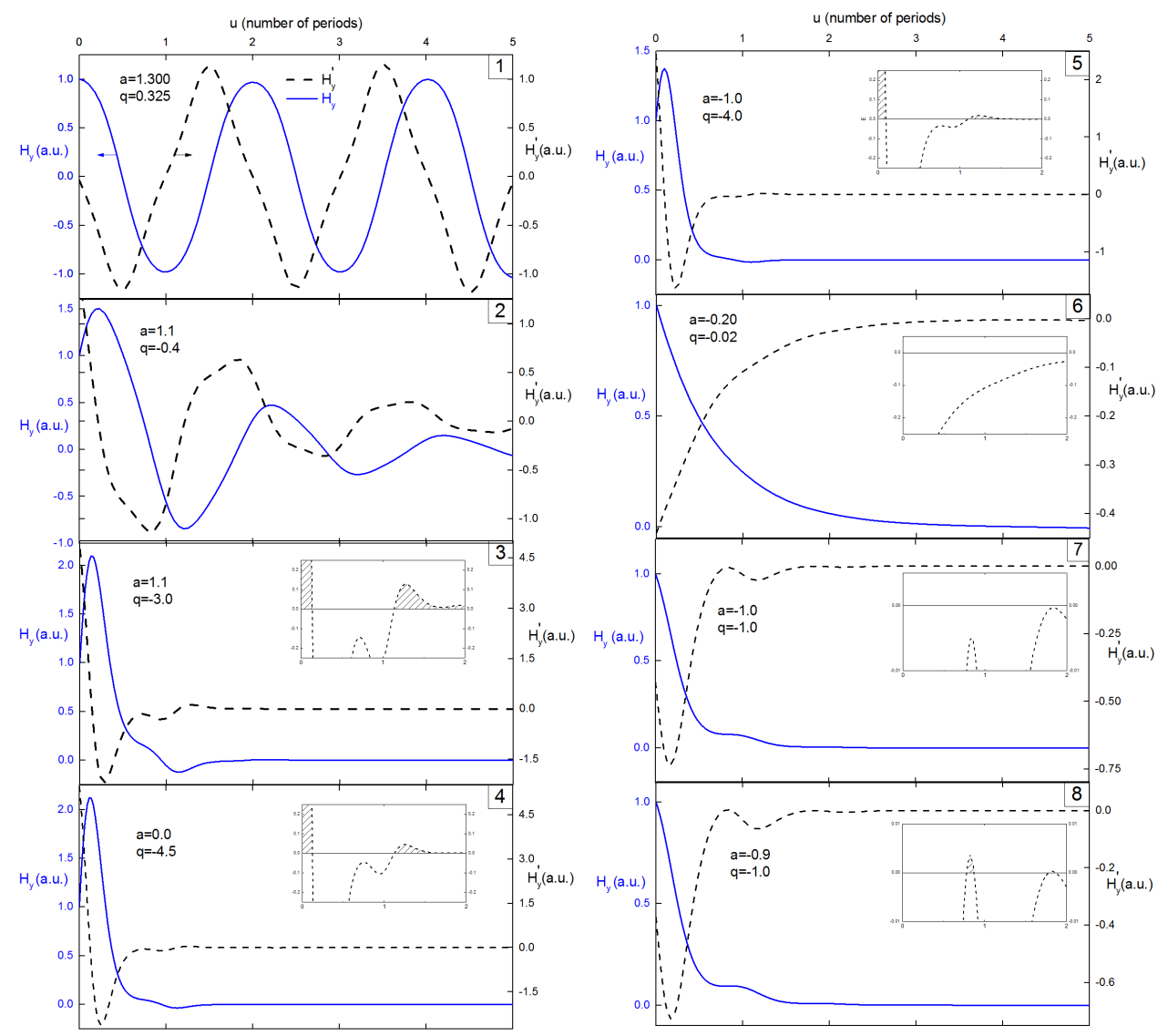

Figure 4. Solutions of the Mathieu's equation plotted for different $(a, q)$-pairs depicted on Fig. 3. The solid blue line corresponds to the magnetic field $H_{y}$, the dashed black line - to its derivative $H_{y}^{\prime}$. A photonic crystal with certain $(a$, $q$ )parameters can support surface waves if its solution is decreasing on the crystal scale and its derivative goes to zero to fulfill the continuity requirement. The inset images a zoomed region of the graph to show that the derivative does go to zero at certain points (graphs $1-4,8$ ) or never goes to zero (graphs 6, 7).

What is the largest value of the external medium dielectric constant $\varepsilon_{1}$ achievable if $\Delta \varepsilon$ is limited by the material properties? To answer this question, we need to use instead of eq. (17) an asymptotic description of the same characteristic curve for large negative $a$ values. This question is not completely trivial, but looking e.g., into Langer's analysis [25] of the Mathieu's equation solutions when at least one of the parameters $a, q$ is large, we found an estimation of the type $a=-|2 q|+2 \sqrt{|q|}+O(\ln |q|)$ (see his eqs. (6.3) and (7.10) and note the difference in notation, $a=\Delta, 2 q=\Omega$, in his paper), which attests that asymptotically $\lim _{a \rightarrow-\infty}(|2 q| / a)=-1$, and also this is known that "no characteristic values exist when $|a| \geq|2 q|$ " (p. 690). From this and eq. (15), we immediately infer that surface electromagnetic waves cannot be obtained if $\varepsilon_{1}>\varepsilon_{L}$. This means that the external medium cannot have a refractive index higher than the largest refractive index of the periodic structure. At the same time, for any $\varepsilon_{1}<\varepsilon_{L}$ and large negative $a$, an appropriate photonic crystal supporting long-range plasmon propagation can be designed. However, for too small differences of refractive indices this requires large values of $|a|$ and, consequently, large periods of the structure which might be impossible to realize in practice. 


\section{Conclusions and discussion}

In the present paper, the application of the Mathieu's equation to excitation of surface waves in photonic crystals was presented. We have discussed a design of a multilayer structure coated with a thin metal film to support surface plasmons, and established the necessary requirements for the solutions of the Mathieu's equation describing surface waves. First, such a solution must vanish at the infinity (or say, on the scale of the multilayer structure) which immediately defines that one should work in the instability regions of the Mathieu's equation stability chart. Further, given by eq. (9) the continuity condition of the tangential electric field component across the interface has to be fulfilled.

A limit of a thin metal film was considered. Here eq. (9) reduces to a simple condition that the decaying at infinity solution of the Mathieu's equation should have a zero derivative at the interface. We have briefly analyzed for which pairs $(a, q)$ belonging to the different instability regions on the Mathieu's equation stability diagram this condition can be fulfilled, and for which cannot (Figs. 3, 4). Several examples of a real multilayer structure were given, and limits appearing for design of photonic crystal structures were discussed. These results constitute a base for further development of a similar approach, for example for the cases when the layer thicknesses $d_{L}$ and $d_{S}$ of the photonic crystal are different. Hill's differential equation [23], sometimes named also "generalized Mathieu equation" is appropriate here.

In conclusion, we would like to draw attention to an analogy of surface waves in a multilayer structure to a quantum particle in a periodic potential, which is also described by the Mathieu's equation [26]. Essentially, the Mathieu's equation, if we rewrite it as $-d^{2} u / d y^{2}+(-a+2 q \cos (2 y)) u=0$, can be seen as a particular case of the non-relativistic stationary Schrödinger equation. This equation (with a proper scaling) describes a quantum particle in a periodic potential $V(y)=2|q| \cos (2 y)=V_{0} \cos (2 y)$ with the total energy of the particle $E=a$. For a given potential value, there may be certain values of $a$ (means $E_{n}$ ) for which bounded solutions (eigenstates) can be found. Such bounded solutions describe surface waves, which we are interested in, and they certainly have a zero derivative at $y=0$, $u^{\prime}(0)=0$, due to the potential symmetry. Obviously, there is no eigenstates with the energies $E$ smaller than the minimal potential value $-V_{0}$ and therefore, no bounded solutions. This analogy is an excellent illustration of the problem of plasmon excitation: none of the Mathieu's equations with a negative parameter $a$ such that $a<-2|q|$ is suitable for the excitation of surface waves. Moreover, this reasoning explains well that for small negative $a$, surface waves cannot be excited for $-\frac{a}{2|q|}>1$, which is perfectly in line with the asymptotic behavior of the parameter $a$ mentioned above.

\section{Acknowledgements}

The research has been fulfilled with the partial financial support of Swiss National Science Foundation, grant $\mathrm{N}$ 200021_150161.

\section{References}

[1] E. Yablonovitch, Photonic band-gap structures, J. Opt. Soc. Am. B, Vol. 10, No. 2, 283-295, 1993.

[2] P. Yeh, A. Yariv and C.S. Hong, Electromagnetic propagation in periodic stratified media. I. General theory. J. Opt. Soc. Am., Vol. 67, No. 4, 423-438, 1977.

[3] J.A. Arnaud and A.A.M. Saleh, Guidance of surface waves by multilayer coatings, Appl. Opt., Vol. 13, No. 10, 2343-2345, 1974.

[4] P. Yeh, A.Yariv and A.Y. Cho, Optical surface waves in periodic layered media, Appl. Phys. Lett., Vol. 32, No. 2, 104-105, 1978.

[5] W.M. Robertson and M.S. May, Surface electromagnetic waves on one-dimensional photonic band gap arrays, Appl. Phys. Lett., Vol. 74, No. 13, 1800-1802, 1999.

[6] A. Shinn and W.M. Robertson, Surface plasmon-like sensor based on surface electromagnetic waves in a photonic band-gap material, Sensor Actuat. B-Chem., Vol. 105, 360-364, 2005.

[7] V.N. Konopsky and E.V. Alieva, Photonic crystal surface waves for optical biosensors, Anal. Chem., Vol. 79, No. 12, 4729-4735, 2007.

[8] V.N. Konopsky, and E.V. Alieva, A biosensor based on photonic crystal surface waves with an independent registration of the liquid refractive index, Biosens. Bioelectron., Vol. 25, 1212-1216, 2010.

[9] Y. Guo, J.Y. Ye, C. Divin, B. Huang, T.P. Thomas, J.R. Baker and T.B. Norris, Real-time biomolecular binding detection using a sensitive photonic crystal biosensor, Anal. Chem. Vol. 82, No. 12, 5211-5218, 2010 .

[10] P. Rivolo, F. Michelotti, F. Frascella, G. Digregorio, P. Mandracci, L. Dominici, F. Giorgis and E. Descrovi, Real time secondary antibody detection by means of silicon-based multilayers sustaining Bloch surface waves, Sensor Actuat. B-Chem., Vol. 161, $1046-1052,2012$.

[11] A. Farmer, A.C. Friedli, S.M. Wright, W.M. Robertson, Biosensing using surface electromagnetic waves in photonic band gap multilayers, Sensor Actuat. B-Chem., Vol. 173, 79-84, 2012.

[12] V.N. Konopsky, D.V. Basmanov, E.V. Alieva, S.K. Sekatskii and G. Dietler, Size-dependent hydrogen uptake behavior of $\mathrm{Pd}$ nanoparticles revealed by 
photonic crystal surface waves, Appl. Phys. Lett., Vol. 100, 83-108, 2012.

[13] V.N. Konopsky, T. Karakouz, E. V. Alieva, C. Vicario, S. K. Sekatskii and G. Dietler, Photonic Crystal Biosensor based on Optical Surface Waves, Sensors, Vol.13, 2566 - 2578, 2013.

[14] B.N. Zaitsev, F. Benedetti, A.G. Mikhaylov, D.V. Korneev, S. K. Sekatskii, T. Karakouz, P.A. Belavin, N.A. Netesova, E.V. Protopopova, S.N. Konovalova, G. Dietler and V.B. Loktev. Force-induced globulecoil transition in Laminin Binding Protein and its role for viral - cell membrane fusion, J. Mol. Recognit., Vol. 27, No. 12, 727 - 738, 2014.

[15] A. Sinibaldi, N. Danz, E. Descrovi, P. Munzert, U. Schulz, F. Sonntag, L. Dominici and F. Michelotti, Direct comparison of the performance of Bloch surface wave and surface plasmon polariton sensors, Sensor Actuat. B-Chem., Vol. 174, 292-298, 2012.

[16] V.N. Konopsky and E.V. Alieva, Long-range propagation of plasmon polaritons in a thin metal film on a one-dimensional photonic crystal surface, Phys. Rev. Lett., Vol. 97, No. 25, 253904, 2006.

[17] S.A. Maier, Plasmonics: Fundamentals and Applications, Springer, New York, 2007.

[18] D. Sarid, Long-Range Surface-Plasma Waves on Very Thin Metal Films, Phys. Rev. Lett., Vol. 47, No. 26, 1927-1930, 1981.

[19] J. J. Burke, G. I. Stegeman, and T. Tamir, Surfacepolariton-like waves guided by thin, lossy metal films, Phys. Rev. B, Vol. 33, No. 3, 221-223, 1986

[20] V.N. Konopsky, D.V. Basmanov, E.A. Alieva, D.I. Dolgy, E.D. Olshansky, S.K. Sekatskii and G. Dietler, Registration of long-range surface plasmon resonance by angle-scattering feedback and its implementation for optical hydrogen sensing, New J. Phys., Vol. 11, 063049, 2009.

[21] E.V. Alieva, V.N. Konopsky, D.V. Basmanov, S.K. Sekatskii, and G. Dietler, Blue surface plasmon propagation along thin gold film - gas interface and its use for sensitive nitrogen dioxide detection, Opt. Commun., Vol. 309, 148 - 152, 2013.

[22] V.N. Konopsky, Plasmon-polariton waves in nanofilms on one-dimensional photonic crystal surfaces, New J. Phys., Vol. 12, 093006, 2010.

[23] N.W. McLachlan, Theory and application of Mathieu functions, Dover, New York, 1964.

[24] J.A. Stratton, Electromagnetic theory, McGraw-Hill, New York, 1941, p. 360.

[25] R.E. Langer, The solutions of Mathieu equation with a complex variable and at least one parameter large, Trans. Am. Math. Soc., Vol. 36, No. 3, 637-695, 1934.
[26] M.J. Richardson, Solution of the Mathieu Equation in the WKB Approximation, Am. J. Phys., Vol. 39, 560565,1971 . 